
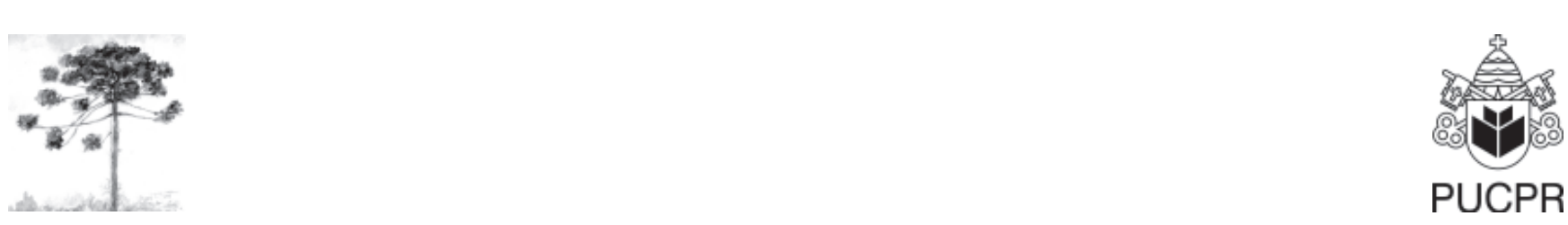

\title{
RELAÇÃO ENTRE ÁREA DE SUPERFÍCIE DO SUBSTRATO E ABUNDÂNCIA DE DUAS ALGAS VERDES FILAMENTOSAS EM UM RIACHO DA REGIÃO CENTRO-SUL DO ESTADO DO PARANÁ
}

\author{
Relationship between surface area of substrate and abundance of two filamentous \\ green algae in a stream of Mid-Southern region of Parana State
}

\section{Rogério Antonio Krupek}

Faculdade Guairacá, Departamento de Biologia, Guarapuava, PR - Brasil, e-mail: rogeriokrupek@yahoo.com.br

\section{Resumo}

A relação entre a área de superfície de substratos rochosos e a abundância de algas bentônicas foi avaliada com o objetivo de encontrar possíveis relações e/ou associações entre estes. O estudo de campo foi desenvolvido em um riacho localizado no município de Guarapuava, região Centro-Sul do Estado do Paraná. A área de superfície das rochas foram calculadas com base em medidas dimensionais simples (comprimento, altura e largura) aplicadas à fórmula proposta por Graham et al. (1988). A abundância das espécies de algas bentônicas foi avaliada a partir de análise visual com o auxílio de um observador subaquático. As análises demonstraram que houve relação significativa entre o tamanho dos substratos e as variáveis biológicas avaliadas. De modo geral, as algas bentônicas foram mais comuns em rochas de menor tamanho, entretanto, as características particulares de cada espécie foram importantes na utilização do substrato. Apesar de muitas variáveis estarem envolvidas na distribuição espacial de algas bentônicas em riachos, este trabalho ressaltou a importância do substrato para esses organismos.

Palavras-chave: Área de superfície; Algas bentônicas; Substrato; Riachos.

\section{Abstract}

The relationship between surface area of stones substrates and abundance of benthic algae were evaluated with aim find possible relations and/or association these. The sampling studies were developed in a stream located in the municipality of Guarapuava, mid-southern region of Paraná 
state. The surface area of stones were calculated based in measured dimensional simple (length, width and height) using to the formula suggest Graham et al. (1988). Species abundance of benthic algae was estimated visually with a Plexiglas viewbox. Analyses demonstrated significant relationships among size of substratum and biological variables. In general, benthic algae were more presents in tiny stones, however the particular characteristics of each species was important in the use of the substratum. Although many variables to be involved in the spatial distribution of benthic algae in streams, this work stand out the importance of the substratum for these organisms.

Keywords: Surface área; Benthic algae; Substrate; Streams.

\section{INTRODUÇÃO}

A distribuição de organismos bentônicos é correlacionada com a variabilidade do ambiente em uma variedade de escalas (1). A heterogeneidade espacial é uma característica estrutural de sistemas ecológicos e pode ser definida geralmente como a complexidade e variabilidade de um sistema próprio no espaço (2).

Algas são frequentemente influenciadas em uma grande extensão pela variação local ou em pequena escala, resultando em uma distribuição em mosaico (3). As algas compreendem o grupo de organismos mais variável com relação a suas estratégias de vida e estas são muito pouco referenciadas quanto à sua distribuição específica em ambientes lóticos (4).

As oportunidades oferecidas pelos substratos de diferentes tamanhos podem afetar a quantidade e a qualidade das algas aderidas (5). Apesar da potencial importância do tamanho do substrato para a distribuição e abundância de algas bentônicas, esta variável ainda apresenta evidências ainda contraditórias (5).

A heterogeneidade em pequena escala é um fator difícil de medir, especialmente em escala de rochas individuais (1). Muitos dos métodos desenvolvidos requerem equipamentos ou softwares especializados $(6,7,8)$, o que dificulta a realização de tais experimentos. A utilização de métodos mais simples de medição da superfície de rochas, apesar de não apresentar a mesma precisão de outros métodos, apresenta grande aplicabilidade e praticidade de medidas em múltiplos substratos (1).
Com base no postulado acima, o objetivo deste trabalho é procurar estabelecer relações entre a área de superfície de diferentes tamanhos de substratos rochosos, a partir de medidas dimensionais simples, com a riqueza e abundância de algas bentônicas em um riacho da região CentroSul do Estado do Paraná.

\section{MATERIAL E MÉTODOS}

O riacho estudado encontra-se no município de Guarapuava (2523"26"S, 5127"15"W), região Centro-Sul do Estado do Paraná. O segmento avaliado, com uma extensão de 10 metros apresenta incidência luminosa alta em todo o trecho (ausência de cobertura marginal), evitando desta forma qualquerinfluência determinada por variações da irradiância. O riacho estudado possui largura média de 1,20 metros, sendo seu leito composto predominantemente de substrato rochoso, de várias classes de tamanho. As amostragens foram realizadas no dia 18 de abril de 2007.

Neste trecho foram selecionadas aleatoriamente 30 rochas de diferentes tamanhos que apresentavam visível crescimento de algas bentônicas (algas visíveis ao olho desarmado (9). Em cada uma destas, foram tomadas medidas de riqueza (número de espécie) e abundância (cobertura percentual) das algas bentônicas. As medidas foram realizadas visualmente com o auxílio de um observador subaquático com fundo de vidro. Amostras de cada uma das espécies de macroalgas foram retiradas para posterior identificação. 
Posteriormente, para cada uma das rochas foram tomadas medidas de comprimento, largura e altura, conforme sua localização no leito do riacho. Todas as medidas foram tomadas com auxílio de uma fita métrica. Estas medidas foram posteriormente utilizadas para calcular a área de superfície de cada uma das rochas. Para tanto, foi utilizada a fórmula proposta por Graham et al. (10): área de superfície $=1.15(\mathrm{LW}+\mathrm{LH}+\mathrm{WH})$, onde $\mathrm{L}=$ comprimento, $\mathrm{W}=$ largura e $\mathrm{H}=$ altura. Esta equação é baseada na fórmula para área de superfície de uma esfera, entretanto, foi modificada para a forma mais realística das rochas a partir da incorporação de três diferentes diâmetros. O resultado é a área de superfície de uma rocha arredondada. Apesar desta medida não representar com exatidão a área de superfície de uma rocha, esta apresenta algumas vantagens com relação a outros métodos que apesar de mais precisos são mais dispendiosos e de difícil aplicabilidade: a) rapidez e facilidade de aplicabilidade das medidas; b) praticidade de medidas em múltiplos substratos; e c) medidas facilmente interpretáveis.

Um número igual de rochas $(\mathrm{n}=30) \mathrm{sem}$ algas (designado controle) foi aleatoriamente amostrado para avaliar possíveis diferenças com relação ao tamanho dos substratos em comparação com aquelas com algas.

As variáveis foram logaritimizadas a fim de normalizar as distribuições e homogeneizar as variâncias. As diferenças entre o tamanho das rochas com 'presença de algas' e 'controle' foram determinadas pelo test t de Student. A relação entre riqueza e abundância de espécies de macroalgas com a área de superfície dos substratos foi avaliada a partir do coeficiente de correlação $r$ de Pearson. Todos os testes estatísticos foram realizados com auxílio do pacote estatístico Statistica 5.0.

\section{RESULTADOS}

De modo geral, o tamanho das partículas de substrato analisadas apresentou-se relativamente pequeno. A área de superfície variou de 42 a $601,46 \mathrm{~cm}^{2}(x=165,8 \pm 112,2)$, o comprimento de 4 a $17 \mathrm{~cm}(x=8,9 \pm 2,9)$, a largura de 3 a $14 \mathrm{~cm}$ $(x=6,0 \pm 2,4)$ e a altura variou de 2 a $12 \mathrm{~cm}$ $(x=5,3 \pm 2,4)$.

Stigeoclonium amoenum e Spirogyra sp. foram as espécies de algas bentônicas ocorrentes no riacho amostrado. Estas duas algas verdes são comumente encontradas em riachos de regiões tropicais, sendo que elas já foram descritas como ocorrendo em ambientes lóticos de várias regiões do Brasil.

O teste t de Student demonstrou que as rochas com presença de algas e o controle (sem algas) apresentaram diferenças significativas $(\mathrm{t}=$ $3,90, \mathrm{p}<0,001)$. As rochas com algas apresentaram área de superfície menor $\left(\mathrm{x}=119,4 \mathrm{~cm}^{2}\right)$ que aquelas sem algas $\left(x=220,7 \mathrm{~cm}^{2}\right)$ (Figura 1$)$.

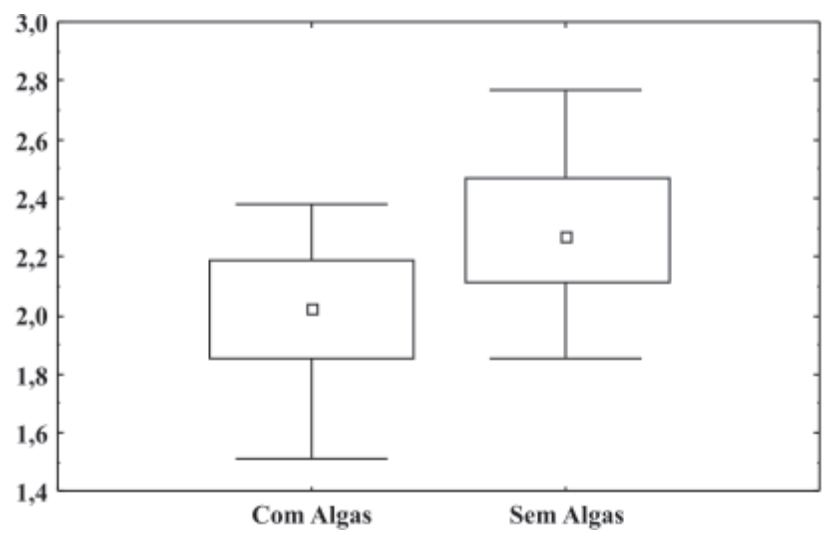

FIGURA 1 - Área de superfície das rochas amostradas "com algas" e "sem algas"

As análises de correlação demonstraram que não houve correlação significativa entre área de superfície do substrato e abundância (cobertura percentual) de espécies $(\mathrm{r}=-0,332, \mathrm{p}=0,07)$ (Figura 2a). Entretanto, as espécies em separado foram registradas correlações significativas: positivas com Stigeoclonium amoenum $(\mathrm{r}=0,444, p<0,05)$, muito pouco frequente e com abundância extremamente baixa (Figura 2b). Negativa com Spirogyra sp. (r=$0,415, \mathrm{p}<0,05)$ que apresentou alta frequência e abundância (Figura 2c). 


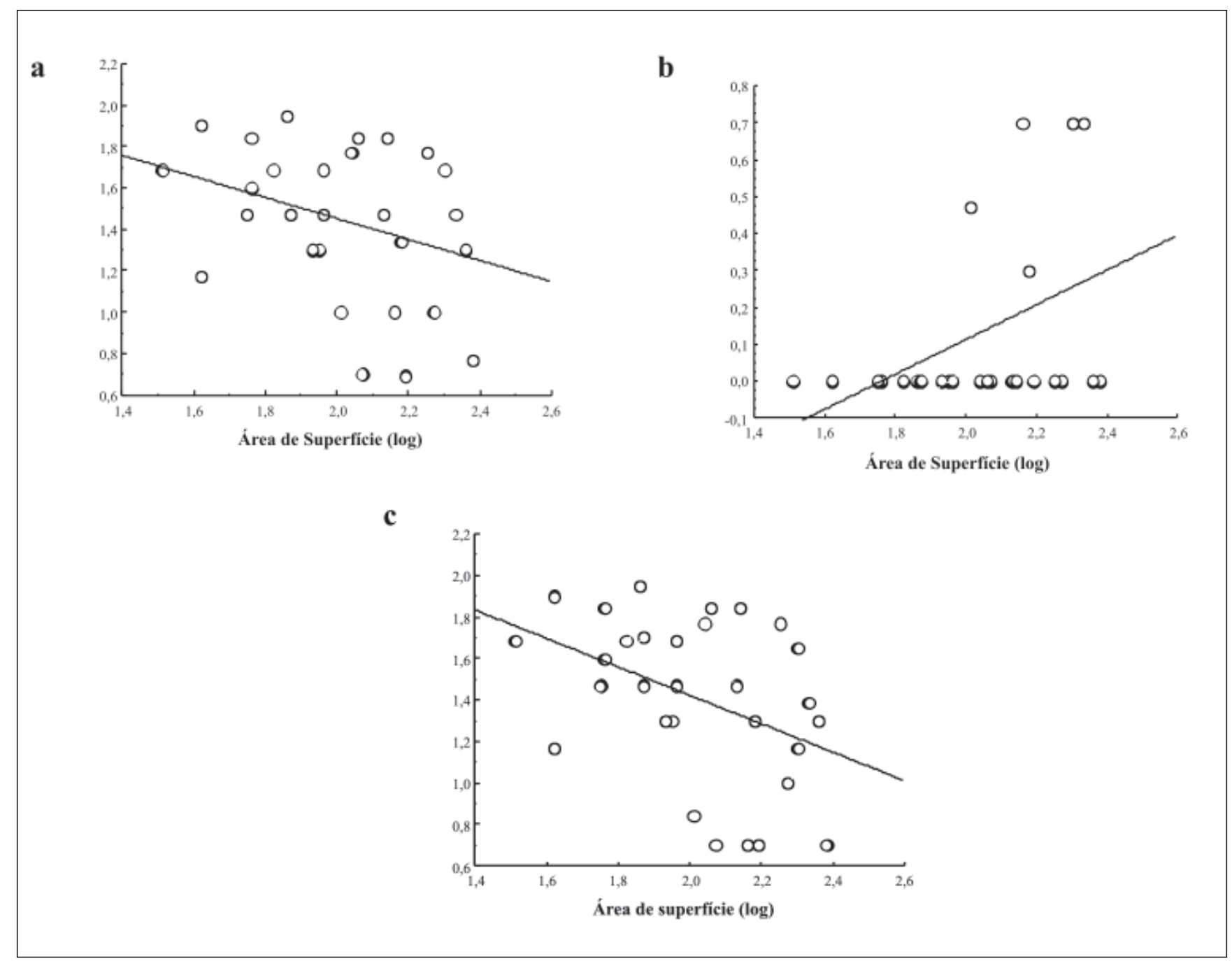

FIGURA 2 - Relação entre área de superfície dos substratos e abundância de espécies (a), abundância de $S$. amoenum (b) e abundância de Spirogyra sp. (c) no riacho estudado

\section{DISCUSSÃO}

Neste segmento de riacho estudado, a abundância de espécies de algas bentônicas ocorrentes apresentou algumas relações com o tamanho dos substratos disponíveis. Relações significativas entre estes organismos e o tipo de substrato têm sido observadas em riachos do Brasil e do mundo (1115). O tamanho das partículas do substrato utilizado por algas bentônicas tem sido descrito como um importante fator de sua distribuição espacial e temporal em riachos $(16,5)$. Segundo Downes et al. (17), macroalgas e briófitas são mais abundantes em grandes rochas que em pequenas, sendo este padrão consistente com aquele encontrado em todo o mundo. Este padrão é dirigido, em ultima instância, pelo postulado de que grandes rochas são mais estáveis, sendo menos provável que sejam carregadas pela correnteza ou coberta por sedimentos (17). Da mesma forma, vários autores têm descrito um aumento na cobertura do perifiton com o consequente aumento do tamanho do substrato (1821). Neste estudo, entretanto, as espécies de algas bentônicas ocorrentes encontraram-se predominantemente em substratos de menor tamanho quando comparado com aqueles que não ocorreram algas aderidas ("controle").

De um modo geral, a ocorrência de substratos de tamanho relativamente pequeno no segmento avaliado e as características particulares das algas presentes no riacho podem ter relevância e explicar a preferência por substratos menores, como mostrado pelos resultados das análises de correlação. 
Estudos que citam relações positivas entre algas bentônicas e o tamanho do substrato fazem referência a grandes substratos, assim como matacão e rocha contínua $(11,15)$. Estes realmente proporcionam maior estabilidade dentro de um ambiente extremamente dinâmico e sujeito a constantes mudanças, características de ambientes de águas correntes. Essa característica particular desses grandes substratos permite às algas bentônicas, uma região, ao mesmo tempo, estável e com um amplo espaço físico, suficiente para a colonização e desenvolvimento de uma comunidade abundante e diversa. Substratos de menor tamanho, por outro lado, geralmente são carregados quando ocorrem eventos que levam ao aumento demasiado no volume d'água, com um correspondente aumento na vasão. Ao longo do tempo, estas pequenas partículas de substrato se acumulam em regiões menos sujeitas a estas ações abrasivas da água, assim como regiões próximas à margem ou em remansos (22). De um modo geral, a ocorrência de algas no riacho estudado foi visivelmente maior nas regiões marginais do que no centro ou na corredeira propriamente dita, corroborando com o postulado acima. Desta forma, a associação de fatores diferenciais, assim como localização e tamanho proporcional dos substratos rochosos com as características hidrológicas do riacho, podem estar relacionados com a presença mais evidente de algas bentônicas em pequenas rochas. As características ecológicas dos táxons ocorrentes no riacho amostrado reforçam os conceitos descritos anteriormente, bem como esclarecem os resultados obtidos a partir dos testes de correlação (Figuras 2a-c). Spirogyra sp. é caracterizada como sendo uma alga metafítica, ou seja, encontra-se na zona fótica de rios e riachos, mas comumente não está diretamente aderida ao substrato e sim fracamente agregada e/ou associada com os diferentes substratos em áreas mais protegidas da correnteza (23). Desta forma, considerando que os substratos de menor tamanho estão concentrados nas regiões marginais do riacho estudado, é coerente que a maior abundância de espécimes de Spirogyra sp. estejam concentradas nesta área.

A relação positiva entre área de superfície do substrato e abundância de Stigeoclonium amoenum, considerando suas características particulares, está consistentemente de acordo com o argumento acima exposto. $S$. amoenum apresenta um sistema de fixação composto de rizoides. Este é relativamente bem desenvolvido, o que lhe permite fixar-se mais firmemente ao substrato, permitindo colonizar ambientes com uma diversa combinação de parâmetros ambientais (24). O tamanho do substrato pode influenciar a porcentagem de cobertura de $S$. amoenum. Em um estudo temporal, Branco e Necchi (11) encontraram as maiores abundâncias de $S$. amoenum em substratos de maiores tamanhos. Esta relação foi explicada com base na estabilidade desses substratos, como já comentado acima, sendo que a abundância de $S$. amoenum foi considerada relativamente alta, quando comparada com Chaetophora elegans (11). Entwisle (25) descreveu aspectos do crescimento de Stigeoclonium e citou a relativa baixa abundância em que ocorria nos riachos em que estava presente, apontando, entre os fatos responsáveis, o tipo de substrato presente. Desta forma, podemos concluir que a baixíssima frequência e cobertura percentual de S. amoenum no riacho estudado está intimamente relacionada, entre outros fatores, à disponibilidade de substrato utilizável por esta alga, já que estes apresentaram, de modo geral, pequena área de superfície.

Apesar da já conhecida relação entre o aumento de tamanho do substrato e consequente aumento na abundância de espécies, características particulares de cada espécie ou morfotipos, bem como algumas características do riacho em particular, parecem exercer papel relevante nesse tipo de associação.

\section{REFERÊNCIAS}

1. Bergey EA. Measuring the surface roughness of stream stones. Hydrobiol. 2006;56(3):247-52.

2. LI H, Reynolds JF. A simulation experiment to quantify spatial heterogeneity in categorical maps. Ecology. 1994;75(8):2446-55.

3. Wright KK, Li JL. From continua to patches: examining stream community structure over large environmental gradients. Can J Fish Aquat Scienc. 2002;59(8):1404-17.

4. Komárek O. Spatial autocorrelation and fractal dimension of alga species assemblage in a gravel stream of central Europe. Rev Hydrobiol. 2003;88(3-4):385-96.

5. Cattaneo A, Kerimian T, Roberge M, Marty J. Periphyton distribution and abundance on substrata of different size along a gradient of stream trophy. Hydrobiol. 1997;354(1-3):101-10. 
6. Clifford HF, Gotceitas V, Casey RJ. Roughness and color of artificial substratum particles as possible factors in colonization of stream invertebrates. Hydrobiol. 1989;175(3):89-95.

7. Schimid PE, Tokeshi M, Schimid-Araya JM. Scaling in stream communities. Proceedings of the Royal Society of London, Series B. 2002;269(1):2587-94.

8. Taniguchi H, Tokeshi M. Effects of habitat complexity on benthic assemblages in a variable environment. Freshw Biol. 2004;49(9):1164-78.

9. Sheath RG, Cole KM. Biogeography of stream macroalgae in North America. J Phycol. 1992;28(4):448-60.

10. Graham AA, Mccaughan DJ, Mckee FS. Measurement of surface area of stones. Hydrobiol. 1988;157(1): 85-7.

11. Branco CCZ, Necchi Jr O. Temporal dynamics of two species of Chaetophoraceae (Chlorophyta) in tropical streams of São Paulo State, southeastern Brazil. Rev Bras Bot. 2003;26(2):151-61.

12. Entwisle TJ. Macroalgae in the upper yarra and watts river catchments: distribution and phenology. Aust J Mar Freshw Res. 1990;41(4):505-22.

13. Hynes HBN. The ecology of running waters. Liverpool: Liverpool University Press; 1970.

14. Necchi Jr. O, Branco CCZ, Simões RCG, Branco LHZ. Distribution of stream macroalgae in northwest region of São Paulo State, southeastern Brazil. Hydrobiol. 1995;299(2):219-30.

15. Necchi Jr. O, Moreira JCL. Longitudinal distribution of macroalgae in two tropical lotic ecossystems from southeastern Brazil. Arch. Hydrobiol.1995;135(1):13-28.

16. Bergey EA, Resh VH. Differential response of algae on small streambed substrates to floods. Am Midl Nat. 2006;155(2):270-7.

17. Downes BJ, Entwisle TJ, Reich P. Effects of flow regulation on disturbance frequencies an in-channel bryophytes and macroalgae in some upland streams. River Res Applic. 2003;19(1):27-42.
18. Cazaubon A, Rolland T, Loudiki M. Heterogeneity of periphyton in French Mediterranean rivers. Hydrobiol. 1995:300/ 301(1):105-14.

19. Mcconnel WJ, Sigler WF. Chlorophyll and productivity in a mountain river. Limnol. Oceanogr.1959;4(3):335-51.

20. Tett P, Gallegos C, Kelly MG, Hornberger GM, Cosby BJ. Relationships among substrate, flow, and benthic microalgal pigment density in the Mechums River, Virginia Limnol Oceanogr. 1978;23(1):785-97.

21. Uehlinger U. Spatial and temporal variability of the periphyton biomass in a prealpine river (Necker, Switzerland). Archiv Für Hydrobiol. 1991;123(2):219-37.

22. Gordon ND, McMahon TA, Finlayson BL. Stream hydrology, an introduction for ecologists. Chichester: John Wiley \& Sons; 1992.

23. Stevenson RJ, Bothwell ML, Lowe RL. Editors. Algal ecology: freshwater benthic ecosystems. San Diego: Academic Press; 1996.

24. Branco CCZ, Necchi Jr. O. Microhabitat and morphometric variation of two Chaetophoraceae (Chaetophorales, Chlorophyta) species in tropical streams of southeastern Brazil. Phycol Res. 1998;46(3):169-74.

25. Entwisle TJ. Macroalgae in Yarra River basin: flora and distribution. Proc Royal Soc Victoria. 1989;101:1-76.
Recebido: 05/05/2007

Received: 05/05/2007

Aprovado: 05/11/2007

Approved: 11/05/2007 